\title{
Towards Quantum Communication with more than 4 bits/photon: Near-Perfect Sorting of the Orbital Angular Momentum Modes of Light
}

\author{
Mehul Malik, ${ }^{1,2 *}$ Mohammad Mirhosseini, ${ }^{1}$ Zhimin Shi, ${ }^{1,3}$ Robert W. Boyd ${ }^{1,4}$ \\ ${ }^{1}$ The Institute of Optics, University of Rochester, Rochester, New York 14627 USA \\ 2 Institute for Quantum Optics and Quantum Information (IQOQI), Austrian Academy of Sciences, Boltzmanngasse 3 , \\ A-1090 Vienna, Austria \\ ${ }^{3}$ Department of Physics, University of South Florida, Tampa, Florida 33620, USA \\ ${ }^{4}$ Department of Physics, University of Ottawa, Ottawa, ON K1N 6N5 Canada \\ *mehul.malik@univie.ac.at
}

\begin{abstract}
We demonstrate a method that uses a series of optical transformations to sort the orbital angular momentum and the mutually-unbiased angular position modes of light with a separation efficiency of more than $92 \%$.

(C)2014 Optical Society of America

OCIS codes: (270.0270) Quantum Optics; (060.2605) Free-space optical communication; (050.4865) Opti-

cal vortices;
\end{abstract}

The orbital angular momentum (OAM) modes of light show great promise as a means to extend quantum communication and computation into the high-dimensional regime. OAM modes reside in a discrete, unbounded state space and have the potential to dramatically increase the information capacity of QKD systems. Furthermore, the use a large alphabet increases the tolerance of a QKD system to eavesdropping attacks.

A key capability for the use of OAM modes in communication is the ability to efficiently sort single photons based on their OAM content. Conventionally, projection measurements are used to measure the OAM content of a photon. However, these methods are limited by a success rate of $1 / N$, where $N$ is the number of OAM modes involved in the experiment. A projection measurement is equivalent to asking a photon, "are you in mode $\ell$ ?" However, we would like to ask the question, "which $\ell$ mode are you in?" The first successful implementation of such a single-photon OAM sorter used a series of cascaded Mach-Zehnder interferometers which proves difficult to implement experimentally [1].

Recently, the group of Miles Padgett demonstrated a method that uses a Cartesian to Log-polar transformation to map the azimuthal phase profile of an OAM mode to a tilted planar wavefront, whose tilt is

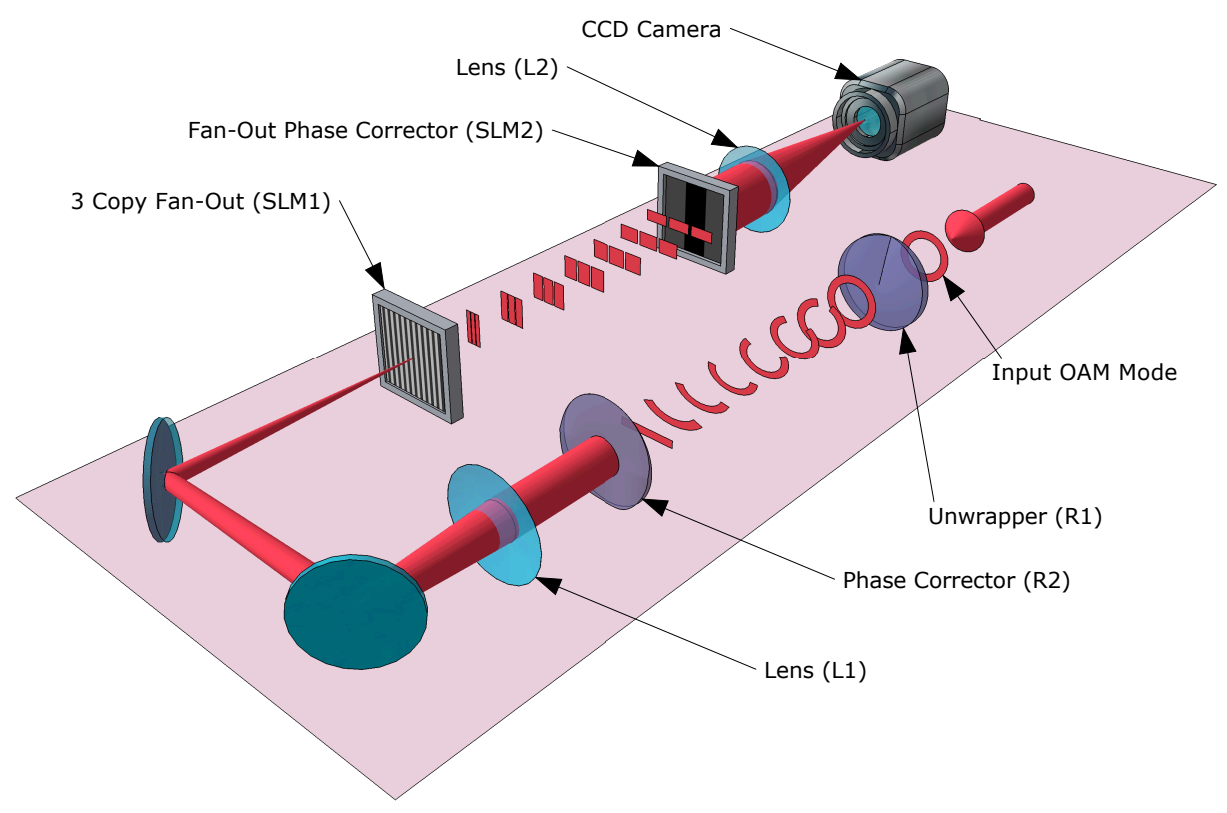

Figure 1: Schematic of the experimental setup used for sorting OAM and ANG modes of light. 


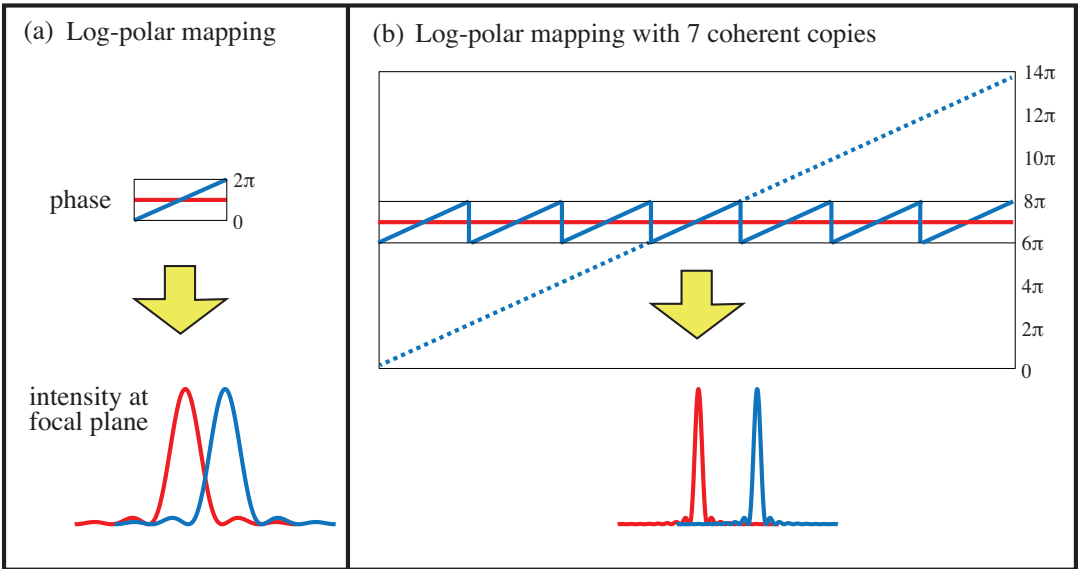

Figure 2: (a) The Log-polar mapping procedure has an inherent cross-talk of $77 \%$ due to the finite size of the mapped plane-wave modes. (b) This cross-talk can be reduced substantially by creating multiple copies of the plane-wave mode and "stitching" them together.

proportional to the OAM quantum number [2]. In this manner, a single photon can be sorted based on the amount of OAM it carries. Unfortunately, due to the finite size of the unwrapped plane-wave mode, there is an inherent amount of inter-modal crosstalk present, which limits this method to a separation efficiency of $77 \%$.

Here we demonstrate an experimental technique that improves the separation efficiency of the previous method to more than $92 \%$ [3]. In order to do this, we first use the unwrapping and phase-correcting elements (R1 and R2 in Fig. 1) described in Ref. [2] to unwrap the OAM mode to a tilted plane-wave mode. Then, we implement a fan-out phase element on a spatial light modulator (SLM1) that creates seven adjacent copies of the transformed plane-wave mode [4]. This results in a plane-wave mode that is not only seven times bigger, but also has a seven times larger phase ramp across it (see Fig. 2). The seven copies are created with a relative phase difference between them that is corrected by another phase element implemented on SLM2. The transformed plane-wave modes are then focused by a lens to spots on a CCD with greater than 92.1\% separation efficiency (Fig. 3a). Theory predicts a separation efficiency as large as $97 \%$. However, this is reduced in the experiment due to the non-ideal behavior of the phase-only SLMs and small misalignments in the system.

We use a similar technique to sort 25 modes in the angular basis (ANG) with a comparable separation
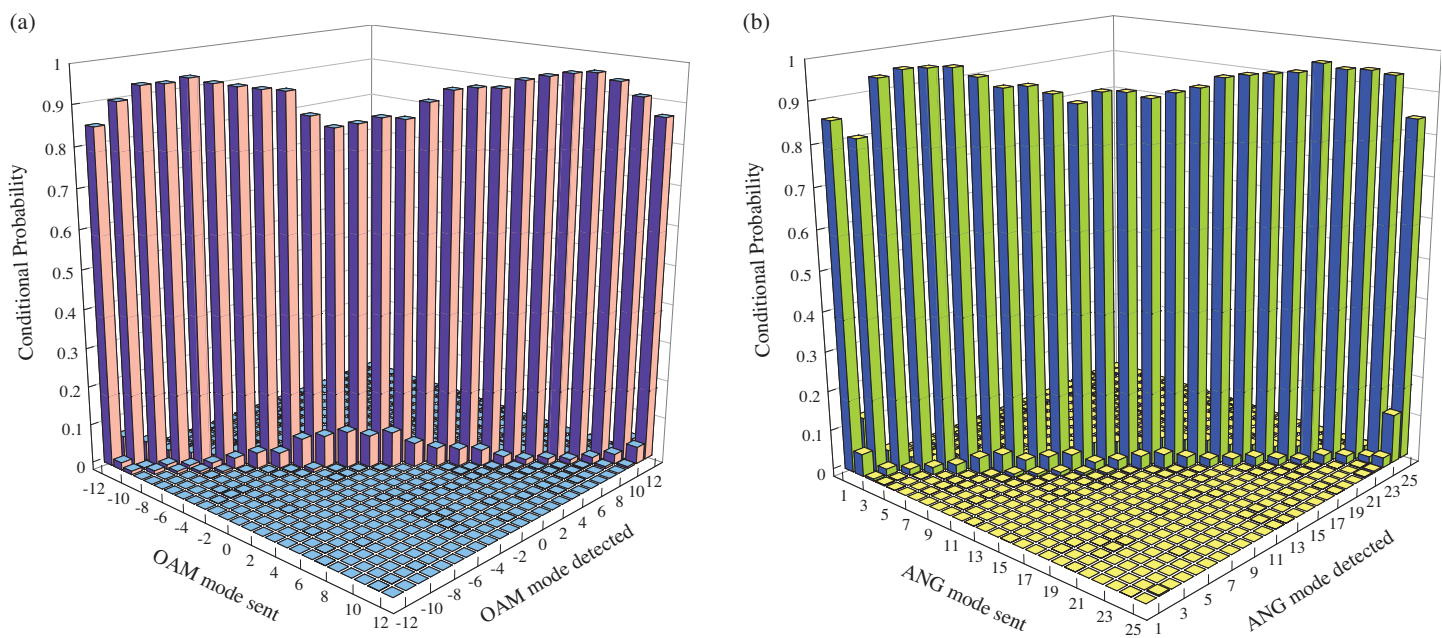

Figure 3: Experimental results showing sorting of (a) 25 OAM and (b) 25 ANG modes. 
efficiency of $92.7 \%$ (Fig. 3b). The ANG basis is made up of modes formed by adding OAM modes with equal weights and a fixed phase relationship between them [5]. This basis mutually unbiased with respect to the OAM basis, which is key for using such high-dimensional modes in quantum key distribution systems. The separation efficiencies measured for the OAM and ANG bases using our method correspond to channel capacities of 4.18 and 4.16 bits per detected photon respectively.

\section{References}

[1] J. Leach, M. J. Padgett, S. M. Barnett, and S. Franke-Arnold, "Measuring the orbital angular momentum of a single photon," Phys. Rev. Lett. (2002).

[2] G. C. G. Berkhout, M. P. J. Lavery, J. Courtial, M. W. Beijersbergen, and M. J. Padgett, "Efficient Sorting of Orbital Angular Momentum States of Light," Phys. Rev. Lett. 105, 153,601 (2010).

[3] M. Mirhosseini, M. Malik, Z. Shi, and R. W. Boyd, "Efficient separation of the orbital angular momentum eigenstates of light," Nat. Commun. 4:2781 (2013).

[4] L. A. Romero and F. M. Dickey, "Theory of optimal beam splitting by phase gratings. I. One-dimensional gratings," J. Opt. Soc. Am. A. 24, 2280-2295 (2007).

[5] M. Malik, M. N. O'Sullivan, B. Rodenburg, M. Mirhosseini, J. Leach, M. P. J. Lavery, M. J. Padgett, and R. W. Boyd, "Influence of atmospheric turbulence on optical communications using orbital angular momentum for encoding," Optics Express 20, 13,195-13,200 (2012). 